\title{
Turbulence: the covariant structure
}

\author{
T. H. Moulden \\ The University of Tennessee Space Institute, USA
}

\begin{abstract}
This paper recalls the covariant structure of the Newtonian theory of fluid mechanics and considers its extension to fluid turbulence. This structure requires that the field equations be covariant under the Newtonian group (that is, under both the Galilean group and the axial group): the dynamics are then consistent with the first axiom of Newtonian continuum thermomechanics. The covariance structure of the Reynolds decomposition under this Newtonian group is the main interest herein.

Keywords: Newtonian fluid mechanics, covariance structure, turbulence.
\end{abstract}

\section{Introductory remarks}

It is generally accepted that constraints must be imposed upon turbulence models to ensure consistency with the covariance structure of Newtonian continuum mechanics. In other words, the mean motion equations and the turbulence model must have a covariance structure that is consistent with the instantaneous Navier Stokes equations. However, some additional constraints must also be imposed upon turbulence models. For example, as was shown in Moulden [1], the compressible flow mean motion equations need not have a unique solution for any arbitrary turbulence model. The theory in Moulden [1] only gave the form required, in norm, to guarantee uniqueness for such turbulence models. No explicit structure for a unique turbulence model is available from within that uniqueness theory; turbulence models must be evaluated individually.

The classical process involved in the construction of a turbulence model can be summarized in the following way. First, a Reynolds decomposition is applied to the field equations to generate a system of mean motion equations. The Reynolds tensor, $\boldsymbol{R}$, as well as other correlation terms, arise from the non-linear terms in the Navier Stokes equations. This Reynolds tensor has a very different structure 
from the Cauchy stress tensor $\mathbf{T}(\mathbf{x}, t)$. The latter follows the dictates of constitutive theory in continuum mechanics while the former must be largely empirical in nature. By definition the Reynolds tensor has the form $\mathcal{R} \equiv \mathcal{E}\left(\rho^{\prime} \mathbf{u} \otimes \mathbf{u}\right)$ (as in equation (E2) below) and is symmetric while the Cauchy stress tensor, $\mathbf{T}$, is only symmetric when body moments and surface couples are absent. This is the case considered below.

Hence, it can be asked: does the Reynolds decomposition process commute with the inherent covariance structure of classical continuum thermomechanics? These two, very distinct, theoretical developments need to be woven together as the theory evolves. Studies on the covariant structure for continuum mechanics go back to Noll [2] and Green and Rivlin [3] and that history need not be repeated herein. The important finding of Green and Rivlin [3] was that Galilean covariance of the energy equation only holds for flow variables which satisfy the linear momentum equation. The present study is based upon the generalized presentation in Moulden [4].

The philosophical stance adopted herein, in conformity with Green and Rivlin [3], implicitly relates the ideas of Newton with those of Leibniz, Joule, Mayer and Helmholtz. To start, there is the first axiom of Newton which provides the underlying invariance structure of classical mechanics; the set of inertial frames have a foundational role in the theory of classical thermomechanics. That Newtonian mechanics does not provide any reason for the Galilean observation of linear force-free motion is well known. The principle of Mach provides an alternative interpretation of that finding of inertia but is still devoid of providing reasons for its existence (see Moulden [5]). The structure of classical thermomechanics must combine these two disparate lines of development: mechanics and thermodynamics. Of course, after the work of Helmholtz, the concept of energy covariance was much enlarged. This observation is particularly true when compared with the primitive vis viva concepts known to Leibniz. At the time of Newton and Leibniz there was little reason to expect thermal energy transfer to be significant for the dynamics of particle motion.

As noted in Speziale [6] the Reynolds tensor need only be Euclidean covariant and so is more general than conformity with Newtonian mechanics per se would require. Since the Galilean group is a subgroup of the Euclidean group, the Reynolds tensor must also be Galilean covariant. For reference, write the Euclidean group, $\mathbb{E}_{u}$, on $\mathbb{R}^{3}$ in the form:

$$
\mathbf{x}^{*}=\mathbf{Q}(t)[\mathbf{x}+\mathbf{d}(t)] ; \quad t^{*}=t+t_{0}
$$

with $\mathbf{d}(t) \in \mathbb{R}^{3}$ an arbitrary time dependent vector and $t_{0} \in \mathbb{R}$ an arbitrary constant time increment. Whence:

$$
\mathbf{v}^{*}=\dot{\mathbf{Q}}(t)[\mathbf{x}+\mathbf{d}(t)]+\mathbf{Q}(t)[\mathbf{v}+\dot{\mathbf{d}}(t)]
$$

Now, the Reynolds decomposition of the velocity field, $\mathbf{v}=\mathbf{V}+\mathbf{u}$, where $\mathbf{V}=\mathcal{E}(\mathbf{v})$, gives the following definitions:

$$
\mathbf{u}^{*}=\mathbf{Q} \mathbf{u} \quad \Rightarrow \quad \mathcal{R}^{*} \equiv \mathcal{E}\left(\rho^{\prime} \mathbf{u}^{*} \otimes \mathbf{u}^{*}\right)=\mathcal{E}\left(\rho^{\prime}(\mathbf{Q u}) \otimes(\mathbf{Q u})=\mathbf{Q} \mathcal{R} \mathbf{Q}^{T}\right.
$$


with $\rho \mapsto \bar{\rho}+\rho^{\prime}$ (since the scalar density is frame indifferent) to show that the Reynolds tensor $\mathcal{R}$ is frame indifferent under the Euclidean group. It is also evident that the equalities $\operatorname{trace}\left(\boldsymbol{\mathcal { R }}^{*}\right)=\operatorname{trace}(\boldsymbol{\mathcal { R }})$ and $\operatorname{det}\left(\boldsymbol{\mathcal { R }}^{*}\right)=\operatorname{det}(\boldsymbol{\mathcal { R }})$ hold under $\mathbb{E}_{u}$. $\mathcal{R}^{*}$ and $\mathcal{R}$ are in the same equivalence class and hence have the same spectrum.

\subsection{The mass invariance constraint}

It is required by Newtonian mechanics that, for any selected body of matter, $\mathcal{B}$, the mass $M(\mathcal{B}) \in \mathbb{R}$ must be an invariant under time translation. This mass is also Galilean covariant. Consequently, $d M(\mathcal{B}) / d t \equiv 0$ as the body, $\mathcal{B}$, moves through spacetime. This latter condition expands as:

$$
M(\mathcal{B})=\int_{\mathcal{B}} d m \equiv \int_{\mathcal{D}_{t}} \rho d V
$$

if $\mathcal{D}_{t}$ is the physical domain occupied by the body $\mathcal{B}$ at time $t$. Next, by the transport theorem:

$$
\frac{d}{d t} M(\mathcal{B})=\frac{d}{d t} \int_{\mathcal{D}_{t}} \rho d V=\int_{\mathcal{D}_{t}}\left[\frac{d \rho}{d t}+\rho \operatorname{div}(\mathbf{v})\right] d V \equiv 0
$$

The localization theorem then provides the usual differential equation:

$$
\frac{\partial \rho}{\partial t}+\operatorname{div}(\rho \mathbf{v})=0
$$

that characterizes mass invariance in every inertial frame.

\subsection{The Galilean group, $\mathbb{G}_{a}$}

Let $\mathbb{O}_{B}=\left\{\varpi_{i}\right\}$ be the set of all Newtonian observers. As required by the first axiom of Newtonian mechanics (extended to apply to continuum mechanics as in Moulden [4] ), there is the group, $\mathbb{G}_{a}$, of transformations (as a subgroup of $\mathbb{E}_{u}$ ) on space $\mathbb{S}_{t}$ at time $t \in \mathbb{T}:$

$$
\mathbf{x}^{*}=\mathbf{Q}_{G}\left[\mathbf{x}+\mathbf{V}_{T} t\right] ; \quad \mathbf{v}^{*}=\mathbf{Q}_{G}\left[\mathbf{v}+\mathbf{V}_{T}\right] ; \quad t^{*}=t
$$

where $\mathbf{Q}_{G} \in \mathbb{S} O_{3}$ is a constant coordinate axis orientation change while the vector $\mathbf{V}_{T} \in \mathbb{R}^{3}$ denotes the constant Galilean boost velocity of the moving coordinate frame $\Lambda^{*} \equiv\left\{\left(\mathbf{x}^{*}, t^{*}\right)\right\}$, fixed to the body $\mathcal{B}$, and relative to the fixed inertial frame $\Lambda_{c} \equiv\{(\mathbf{x}, t)\}$. By inspection, equation (1) is just a restriction of (E). The field equations are inherently indifferent under space and time translation and there is no need to include these features in the group $\mathbb{G}_{a}$ defined in equation (1). If $\mathbf{L}=\partial \mathbf{v} / \partial \mathbf{x}$ is the velocity gradient tensor then $\mathbf{L} \mapsto \mathbf{Q}_{G} \mathbf{L} \mathbf{Q}_{G}^{T}$ under $\mathbb{G}_{a}$. The body force per unit mass, $\mathbf{f}_{B}^{m}$, transforms as a vector: $\mathbf{f}_{B}^{m} \mapsto \mathbf{Q}_{G} \mathbf{f}_{B}^{m}$. The same is true of the stress vector: $\mathbf{t} \mapsto \mathbf{Q}_{G} \mathbf{t}$ so that the stress tensor, $\mathbf{T}$, transforms as $\mathbf{T} \mapsto \mathbf{Q}_{G} \mathbf{T} \mathbf{Q}_{G}^{T}$. Each inertial frame, $\Lambda_{i}$, supports its own observer $\varpi_{i} \in \mathbb{O}_{B}$. Scalars, such as the density $\rho$ and temperature $\theta$ are invariant across inertial frames so that:

$$
\rho^{*}\left(\mathbf{x}^{*}, t^{*}\right)=\rho(\mathbf{x}, t) \quad \text { and } \quad \theta^{*}\left(\mathbf{x}^{*}, t^{*}\right)=\theta(\mathbf{x}, t)
$$


Similarly for the fluid pressure $P(\mathbf{x}, t)$. Note also that $\operatorname{div}^{*}\left(\mathbf{s}^{*}\right)=\operatorname{div}(\mathbf{s})$ for any frame indifferent vector $\mathbf{s}$ (as required to show the covariance of equation (M1) across inertial frames). The field equations are written in the $\Lambda^{*}$ frame and then transferred back to the $\Lambda_{c}$ frame though equation (1).

According to equation (1) the observers $\varpi^{*}$ and $\varpi$ move at constant velocity relative to each other but both observers view the same thermomechanics within the Newtonian framework.

\subsection{The axial group, $\mathbb{G}_{z}$}

The transformation that represents the requirement of Newton that rotational motion persist unless impeded by some torque, is given by the group, $\mathbb{G}_{z}$, of rotations:

$$
\mathbf{x}^{*}=\mathbf{Q}(t) \mathbf{x} ; \quad \mathbf{Q}(t)=\exp [\mathbf{Z} t] ; \quad t^{*}=t
$$

with $\mathbf{Z} \equiv \mathbf{Q}^{T} \dot{\mathbf{Q}} \in \mathbb{S}_{\text {sk }}^{3}$ a constant skew symmetric tensor whose axial vector is denoted by $\mathbf{r} \in \mathbb{R}^{3}$. Thermomechanics must be covariant under $\mathbb{G}_{z}$ to satisfy Newton's requirement that: "A spinning hoop $\cdots$ does not cease to rotate, except insofar as it is retarded by the air", as stated following axiom I in Newton [7]. Equation (2) implies that:

$$
\mathbf{v}^{*}=\dot{\mathbf{Q}} \mathbf{x}+\mathbf{Q} \mathbf{v}=\mathbf{Q}[\mathbf{Z} \mathbf{x}+\mathbf{v}]
$$

and that:

$$
\mathbf{a}^{*}=\dot{\mathbf{Q}}[\mathbf{Z} \mathbf{x}+\mathbf{v}]+\mathbf{Q}[\mathbf{Z} \mathbf{v}+\mathbf{a}]
$$

Since the angular momentum equation only concerns the symmetry of the Cauchy stress tensor, as in Moulden [4], there is little need to include it in the present discussion.

\subsection{Covariant requirements}

Newtonian continuum mechanics must be covariant under the Newtonian group, that is: the Newtonian equations of continuum thermomechanics must be covariant under both the Galilean and axial groups: the field equations must have the same form in all inertial frames. This must be true for all flow conditions: the fluctuating quantities associated with turbulent flow do not countermand this requirement. The starting point for a study of covariance, in the present context, is the total energy equation stated in equations (3) and (3a) below; as well as the mass invariance constraint expressed as the condition $M(\mathcal{B})=$ constant. The latter is trivially true and needs no further comment beyond the discussion in section 1.1 above.

\section{The Newtonian covariant structure}

The concepts involved here just require the field equations to be covariant across changes in inertial frames in conformity with Newtonian continuum mechanics. 
It was shown by Green and Rivlin [3] that the total energy equation only enjoys this covariance provided that the linear momentum equation holds true. Explicitly, the linear momentum equation follows from covariance under the boost velocity in the Galilean group. This then is the objective of the present paper: what does this covariant structure imply about the nature of the Reynolds stresses in compressible turbulent fluid motion? In summary:

Axiom 2.0: The covariant structure of Newtonian continuum thermomechanics can be stated in the form:

1). The total mass of the Newtonian universe is time invariant.

2). The total energy of the Newtonian universe is time invariant.

3). The field equations that describe items 1). and 2). above must be covariant across all inertial frames. That is, the theory must be covariant under the Newtonian group.

Corollary 2.0: The linear momentum equation holds.

The covariant structure presented in axiom 2.0 and its corollary is reviewed below and then extended to include the classical Reynolds decomposition often adopted to discuss fluid turbulence.

To set the background to the present study, review the basic theory from Moulden [4]. Start the development from the inductive statement:

Axiom 2.1: (Clausius): Total energy invariance

In the inertial frame $\Lambda_{c}=\{(\mathbf{x}, t)\}$ the total energy, $E$, of the entire universe $O O$ is invariant under time translation.

and requires that $\mathcal{A}(0, t)=0$ for all time. Here, the definitions, are:

$$
\mathcal{A}(\mathcal{D}, t) \equiv \frac{d E}{d t}=\int_{\mathcal{D}} \rho\left[\frac{d e}{d t}+\langle\mathbf{v}, \mathbf{a}\rangle\right] d V
$$

if $e(\mathbf{x}, t)$ is the specific internal energy and $\mathbf{a}=d \mathbf{v} / d t$ the local acceleration vector for motion in the domain $\mathcal{D}$ occupied by body $\mathcal{B}$. For $\mathcal{A}(\infty, t)$ to satisfy item (2) above there must be energy transfer across the boundary, $\partial \mathcal{D}$, of the domain $\mathcal{D}$ given by the quantities $\mathrm{P}(\mathcal{D}, t)$ and $\mathrm{Q}(\mathcal{D}, t)$. It is assumed that both $\mathrm{P}(0, t)$ and $\mathrm{Q}(00, t)$ vanish identically. It is also assumed here that body moments and surface couples are neglected, and that $\mathbf{f}_{B}^{m}$ denotes the body force per unit mass. Then there is:

$$
\mathrm{P}(\mathcal{D}, t)=\int_{\mathcal{D}} \rho\left\langle\mathbf{f}_{B}^{m}, \mathbf{v}\right\rangle d V+\int_{\partial \mathcal{D}}\langle\mathbf{t}, \mathbf{v}\rangle d A ; \quad \mathrm{Q}(\mathcal{D}, t)=-\int_{\partial \mathcal{D}}\langle\mathbf{q}, \mathbf{n}\rangle d A
$$

provided that $\mathbf{t}(\mathbf{x}, t)$ is the stress vector (related to the Cauchy stress tensor by $\mathbf{T n}=\mathbf{t}$ for outward unit normal $\mathbf{n})$ and $\mathbf{q}(\mathbf{x}, t)$ denotes the heat flux vector with $\mathbf{q} \mapsto \mathbf{Q}_{G} \mathbf{q}$ under $\mathbb{G}_{a}$. Then, utilizing the divergence theorem there is, in place of equations $(4 a, b)$, the system:

$$
\mathrm{P}(\mathcal{D}, t)=\int_{\mathcal{D}}\left[\rho\left\langle\mathbf{f}_{B}^{m}, \mathbf{v}\right\rangle+\operatorname{div}\left(\mathbf{T}^{T} \mathbf{v}\right)\right] d V ; \quad \mathrm{Q}(\mathcal{D}, t)=-\int_{\mathcal{D}} \operatorname{div}(\mathbf{q}) d V
$$

Assume in what follows that the Cauchy stress tensor is symmetric so that:

$$
\operatorname{div}\left(\mathbf{T}^{T} \mathbf{v}\right)=\langle\operatorname{div}(\mathbf{T}), \mathbf{v}\rangle+\operatorname{trace}(\mathbf{T L})
$$


This symmetry assumption is consistent with the request of covariance under the axial group $\mathbb{G}_{z}$ as shown in Green and Rivlin [3] as well as Moulden [4]. The explicit form of the Cauchy stress tensor is not required (but the above noted transformation $\mathbf{T}^{*}=\mathbf{Q}_{G} \mathbf{T} \mathbf{Q}_{G}^{T}$ is assumed to hold).

Heat sources, and radiant heat transfer, are not included in the definition of $\mathrm{Q}(\mathcal{D}, t)$ given above. No fluctuations in the body force will be considered in the present formulation.

The first principle of thermomechanics requires, using equations (3) and (4c,d), that the equality:

$$
\mathcal{A}^{*}\left(\mathcal{D}^{*}, t^{*}\right)=\mathrm{P}^{*}\left(\mathcal{D}^{*}, t^{*}\right)+\mathrm{Q}^{*}\left(\mathcal{D}^{*}, t^{*}\right)
$$

must hold for all fluid motions in the frame $\Lambda^{*}$ (fixed to the body $\mathcal{B}$ and moving at constant velocity, $\mathbf{V}_{T}$, relative to frame $\Lambda_{c}$ ). This implied velocity field may be laminar or if it may be turbulent. All Newtonian observers must find field equations of the same form as equation (3a) when equations $(4 \mathrm{c}, \mathrm{d})$ are included. That is, introduce the quantity $(A)$ from the equation:

$$
\begin{aligned}
(A) & \equiv\left\{\int_{\mathcal{D}}\left[\rho^{*} \frac{d e^{*}}{d t}+\operatorname{div}\left(\mathbf{q}^{*}\right)\right] d V^{*}\right. \\
& \left.-\int_{\mathcal{D}}\left[\left\langle\left(\rho\left(\mathbf{f}_{B}^{m *}-\mathbf{a}^{*}\right)+\operatorname{div}\left(\mathbf{T}^{*}\right)\right), \mathbf{v}^{*}\right\rangle+\operatorname{trace}\left(\mathbf{T}^{*} \mathbf{L}^{*}\right)\right] d V^{*}=0\right\} \\
& \equiv \mathcal{A}\left(\mathcal{D}^{*}, t^{*}\right)-\mathrm{P}^{*}\left(\mathcal{D}^{*}, t^{*}\right)-\mathrm{Q}^{*}\left(\mathcal{D}^{*}, t^{*}\right)
\end{aligned}
$$

with the notation $(A)=\Sigma\left(A_{i}\right)$ adopted to identify the individual terms in equation (5). The required result is, indeed, transparent from equation (5).

\subsection{The Galilean group covariance}

The above development leading to equation (5) can be formalized. The form given by Newton for the first axiom was restricted to point masses subjected to forces only. Such a specification is not adequate for continuum thermomechanics. Hence, start by recalling the first axiom of Newtonian continuum mechanics, the axiom of inertia for continuous thermomechanical bodies (copied from Moulden [4]) as an extension of the first axiom of Newton:

Axiom 2.2: Newton (extended),

Adopt the constraint $\mathbf{2 . 3}$ given below then, the center of mass of body $B$ moves relative to the frame $\Lambda_{c}$, such that:

1). With no angular velocity of frame $\Lambda$ relative to $\Lambda_{c}$ there is:

a). the speed, $|\mathbf{v}|$, is a bounded constant

b). the motion is rectilinear

to define linear inertial motion and the geodesics of spacetime.

2). With no linear displacement of frame $\Lambda$ relative to $\Lambda_{c}$ there is:

$c$ ). the angular speed, $|\omega|$, about a fixed axis through the center of mass of $B$, is a bounded constant. 
The canonical frame, $\Lambda_{c}$, is located at the mass center of this Newtonian universe 0 .

For the motion of a deformable heat conducting continuum body accept the epistemological statement:

Constraint 2.3: Every material body $\mathcal{B}$ moves as a rigid body unless acted upon by forces or by torques or unless thermal energy passes across the surface, $\partial \mathcal{B}$, of $\mathcal{B}$ or between the parts of $\mathcal{B}$. In addition, no heat sources exist in $\mathcal{B}$, nor do electromagnetic fields and thermal radiation act upon $\mathcal{B}$. These constraints imply that the internal energy, $e(\mathcal{B})$, is an invariant of body $\mathcal{B} \prec 0$; where 00 denotes the universal body.

again, as given in Moulden [4]. Hence both linear and angular motion are included in axiom 2.2 so that both the Galilean and axial groups defined above are specified as part of the first axiom of Newtonian continuum mechanics. These two groups relate to disparate properties of the energy equation (3) as outlined below. For example, consider covariance of equation (3a) under the Galilean group, there must be in the transformed frame $\Lambda_{c}$, from equation (5), the equality:

$$
\int_{\mathcal{D}}\left[\left\langle\left(\mathbf{a}-\mathbf{f}_{B}^{m}-\operatorname{div}(\mathbf{T}) / \rho\right), \mathbf{V}_{T}\right\rangle\right] d V=0
$$

since the material derivative $d(\varphi) / d t$ is invariant across inertial frames for any frame invariant scalar $\varphi$. Since the boost velocity $\mathbf{V}_{T}$ is an arbitrary constant vector, there is the equality:

$$
\int_{\mathcal{D}}\left[\mathbf{a}-\mathbf{f}_{B}^{m}-\operatorname{div}(\mathbf{T}) / \rho\right] d V \equiv 0
$$

to recover the global Cauchy (linear momentum) equation as shown in Moulden [4]. With the localization theorem equation (7) reduces to:

$$
\rho \mathbf{a}=\rho \mathbf{f}_{B}^{m}+\operatorname{div}(\mathbf{T})
$$

as the local linear momentum equation of Newtonian continuum mechanics. The symmetry of the Cauchy stress tensor follows directly from the covariance of the field equations under the axial group $\mathbb{G}_{z}$ when body moments and surface couples are absent and need not be repeated herein.

\subsection{Summary of the covariant formulation}

The covariant formulation of the equations of continuum mechanics in the classical Newtonian sense demands (see Green and Rivlin [3] ) that the total energy equation is only covariant under Galilean transformations if the Cauchy equation holds true. That is, the Cauchy equation (the second axiom of Newtonian mechanics for the continuum) is not primitive in the context of classical thermomechanics.

\section{The turbulent flow framework}

The main interest herein is the interaction of the Reynolds decomposition with the covariant structure of Newtonian mechanics outlined above. The first step is 
to combine the Galilean group with the Reynolds decomposition to obtain the standard equations in the current variables. Given the non-linearity of the Navier Stokes equations, it is not directly obvious that these two operations, the Reynolds decomposition and the covariant structure, must commute.

\subsection{The Reynolds decomposition}

Start by introducing the Reynolds decomposition in the standard form for the variables that appear in equation (5):

$\begin{array}{lrrrr}\text { for scalars: } & e \mapsto \bar{e}+e^{\prime} ; & \rho & \mapsto \bar{\rho}+\rho^{\prime} & \\ \text { for vectors: } & \mathbf{a} \mapsto \overline{\mathbf{a}}+\mathbf{a}^{\prime} ; & \mathbf{q} \mapsto \overline{\mathbf{q}}+\mathbf{q}^{\prime} ; & \mathbf{v} \mapsto \overline{\mathbf{v}}+\mathbf{v}^{\prime} \\ \text { for second order tensors: } & \mathbf{L} \mapsto \overline{\mathbf{L}}+\mathbf{L}^{\prime} ; & \mathbf{T} \mapsto \overline{\mathbf{T}}+\mathbf{T}^{\prime}\end{array}$

The above decomposition assumes the linear viscous fluid so that the Cauchy stress tensor only depends upon the velocity gradient:

$$
\mathbf{T}=\mu\left[\mathbf{L}+\mathbf{L}^{T}\right]-\left[P-\mu^{*} \operatorname{trace}(\mathbf{L})\right] \mathbf{I}
$$

and is frame indifferent under the Galilean group. In addition, let $\mathcal{E}$ denote the Reynolds average operation on any given inertial frame. Next, adopt the Galilean group from equation (1) so that:

$$
\mathbf{v}^{*}=\mathbf{Q}_{G}\left[\mathbf{v}+\mathbf{V}_{T}\right] ; \quad \mathbf{a}^{*}=\mathbf{Q}_{G} \mathbf{a} ; \quad t^{*}=t
$$

with $\mathbf{Q}_{G}$ and $\mathbf{V}_{T}$ constants. This spacetime transformation implies that $d^{*}(\cdot) / d t \equiv$ $d(\cdot) / d t$ and that $\partial(\cdot) /\left.\partial x_{i}^{*} \equiv Q_{G}\right|_{i j} \partial(\cdot) / \partial x_{j}$. The time derivative transforms under $\mathbb{G}_{a}$ as: $\partial(\cdot) / \partial t^{*} \equiv \partial(\cdot) / \partial t-\left.Q_{G}\right|_{j i} V_{j} \partial(\cdot) / \partial x_{i}$. Also, it is assumed that the body force per unit mass, $\mathbf{f}_{B}^{m}$, does not have a fluctuating component. If such fluctuations in $\mathbf{f}_{B}^{m}$ were to be included, they would act as a forcing function on the turbulence and additional correlations, over those included below, would be required in the theory. The comment made in section 4 below would then be relevant.

\subsection{The Galilean group covariance}

The individual terms, $\left(A_{i}\right)$, in equation (5) transform under the Reynolds decomposition as:

$$
\begin{aligned}
\mathcal{E} \int_{\mathcal{D}} \rho \frac{d e}{d t} d V & \equiv \int_{\mathcal{D}}\left[\bar{\rho} \frac{d \bar{e}}{d t}+\mathcal{E}\left(\rho^{\prime} \frac{d e^{\prime}}{d t}\right)\right] d V \\
\mathcal{E} \int_{\mathcal{D}} \rho \operatorname{div}(\mathbf{q}) d V & \equiv \int_{\mathcal{D}}\left[\bar{\rho} \operatorname{div}(\overline{\mathbf{q}})+\mathcal{E}\left(\rho^{\prime} \operatorname{div}\left(\mathbf{q}^{\prime}\right)\right)\right] d V \\
\mathcal{E} \int_{\mathcal{D}}\left\langle\rho \mathbf{f}_{B}^{m}, \mathbf{v}\right\rangle d V & \equiv \int_{\mathcal{D}}\left[\left\langle\bar{\rho} \mathbf{f}_{B}^{m}, \overline{\mathbf{v}}\right\rangle+\mathcal{E}\left\langle\rho^{\prime} \mathbf{f}_{B}^{m}, \mathbf{v}^{\prime}\right\rangle\right] d V
\end{aligned}
$$


$\left(A_{4}\right)$

$$
-\mathcal{E} \int_{\mathcal{D}}\langle\rho \mathbf{a}, \mathbf{v}\rangle d V \equiv-\int_{\mathcal{D}}\left[\langle\bar{\rho} \overline{\mathbf{a}}, \overline{\mathbf{v}}\rangle+\mathcal{E}\left\langle\rho^{\prime} \mathbf{a}^{\prime}, \mathbf{v}^{\prime}\right\rangle\right] d V
$$

$$
-\int_{\mathcal{D}} \mathcal{E}\left[\left\langle\rho^{\prime} \mathbf{a}^{\prime}, \overline{\mathbf{v}}\right\rangle+\left\langle\bar{\rho} \mathbf{a}^{\prime}, \mathbf{v}^{\prime}\right\rangle+\left\langle\rho^{\prime} \overline{\mathbf{a}}, \mathbf{v}^{\prime}\right\rangle\right] d V
$$

$$
\mathcal{E} \int_{\mathcal{D}}\langle\operatorname{div}(\mathbf{T}), \mathbf{v}\rangle d V \equiv \int_{\mathcal{D}}\langle\operatorname{div}(\overline{\mathbf{T}}), \overline{\mathbf{v}}\rangle d V+\int_{\mathcal{D}} \mathcal{E}\left\langle\operatorname{div}\left(\mathbf{T}^{\prime}\right), \mathbf{v}^{\prime}\right\rangle d V
$$

$\left(A_{6}\right) \quad \mathcal{E} \int_{\mathcal{D}} \operatorname{trace}(\mathbf{T} \mathbf{L}) d V \equiv \int_{\mathcal{D}} \operatorname{trace}(\overline{\mathbf{T}} \overline{\mathbf{L}}) d V+\int_{\mathcal{D}} \mathcal{E}\left(\operatorname{trace}\left(\mathbf{T}^{\prime} \mathbf{L}^{\prime}\right)\right) d V$

In equation $\left(A_{1}\right)$, the (scalar) internal energy, $e(\mathbf{x}, t)$, is taken to be a property of the fluid material and not of the motion of that material. Hence, the term $d e / d t$ is identical in all inertial frames. Apply the Reynolds decomposition to equations $(9 a, b)$ to find:

$$
\overline{\mathbf{v}}^{*}=\mathbf{Q}_{G}\left[\overline{\mathbf{v}}+\mathbf{V}_{T}\right] ; \quad \mathbf{v}^{*}=\mathbf{Q}_{G} \mathbf{v}^{\prime} ; \quad \overline{\mathbf{a}}^{*}=\mathbf{Q}_{G} \overline{\mathbf{a}} ; \quad \mathbf{a}^{\prime *}=\mathbf{Q}_{G} \mathbf{a}^{\prime}
$$

and only influences the kinematic components of the equations. There is also, for $\mathbf{L}$ the transformations: $\overline{\mathbf{L}} \mapsto \mathbf{Q}_{G} \overline{\mathbf{L}} \mathbf{Q}_{G}^{T}$ and $\mathbf{L}^{\prime} \mapsto \mathbf{Q}_{G} \mathbf{L}^{\prime} \mathbf{Q}_{G}^{T}$ under $\mathbb{G}_{a}$. The Cauchy stress tensor $\mathbf{T}$ is, as noted above, assumed to be frame indifferent so that $\mathbf{T} \mapsto \mathbf{Q}_{G} \mathbf{T} \mathbf{Q}_{G}^{T}$ under $\mathbb{G}_{a}$ and no $\mathbf{V}_{T}$ term is generated. The Reynolds tensor $\mathcal{R}\left(\rho^{\prime} \mathbf{v}^{\prime} \otimes \mathbf{v}^{\prime}\right)$ transforms as: $\mathcal{R}^{*}=\mathbf{Q}_{G} \mathcal{R} \mathbf{Q}_{G}^{T}$ as a restriction of equation (E2) to the Galilean group.

Consider the above decompositions of (A) into the components $\left(A_{i}\right)$ of equation (5). Application of the Galilean group gives the following transformations of the $\left(A_{i}\right)$ :

$$
\mathcal{E} \int_{\mathcal{D}} \rho \operatorname{div}(\mathbf{q}) d V \mapsto \int_{\mathcal{D}}\left[\bar{\rho} \operatorname{div}(\overline{\mathbf{q}})+\mathcal{E}\left(\rho^{\prime} \operatorname{div}\left(\mathbf{q}^{\prime}\right)\right)\right] d V
$$

$$
\begin{aligned}
\mathcal{E} \int_{\mathcal{D}} \rho\left\langle\mathbf{f}_{B}^{m}, \mathbf{v}\right\rangle d V & \mapsto \int_{\mathcal{D}}\left[\bar{\rho}\left\langle\mathbf{f}_{B}^{m}, \overline{\mathbf{v}}\right\rangle+\mathcal{E}\left\langle\rho^{\prime} \mathbf{f}_{B}^{m}, \mathbf{v}^{\prime}\right\rangle\right] d V+\int_{\mathcal{D}} \bar{\rho}\left\langle\mathbf{f}_{B}^{m}, \mathbf{V}_{T}\right\rangle d V \\
-\mathcal{E} \int_{\mathcal{D}}\langle\rho \mathbf{a}, \mathbf{v}\rangle d V & \mapsto-\int_{\mathcal{D}}\left[\langle\bar{\rho} \overline{\mathbf{a}}, \overline{\mathbf{v}}\rangle+\mathcal{E}\left\langle\rho^{\prime} \mathbf{a}^{\prime}, \mathbf{v}^{\prime}\right\rangle\right] d V-\int_{\mathcal{D}}\left\langle\bar{\rho} \overline{\mathbf{a}}, \mathbf{V}_{T}\right\rangle d V \\
& -\int_{\mathcal{D}} \mathcal{E}\left[\left\langle\rho^{\prime} \mathbf{a}^{\prime},\left(\overline{\mathbf{v}}+\mathbf{V}_{T}\right)\right\rangle+\left\langle\bar{\rho} \mathbf{a}^{\prime}, \mathbf{v}^{\prime}\right\rangle+\left\langle\rho^{\prime} \overline{\mathbf{a}}, \mathbf{v}^{\prime}\right\rangle\right] d V
\end{aligned}
$$

$$
\begin{aligned}
\mathcal{E} \int_{\mathcal{D}}\langle\operatorname{div}(\mathbf{T}), \mathbf{v}\rangle d V & \mapsto \int_{\mathcal{D}}\langle\operatorname{div}(\overline{\mathbf{T}}), \overline{\mathbf{v}}\rangle d V \\
& +\int_{\mathcal{D}} \mathcal{E}\left\langle\operatorname{div}\left(\mathbf{T}^{\prime}\right), \mathbf{v}^{\prime}\right\rangle d V+\int_{\mathcal{D}}\left\langle\operatorname{div}(\overline{\mathbf{T}}), \mathbf{V}_{T}\right\rangle d V
\end{aligned}
$$


$\left(A_{6}^{\prime}\right) \quad \mathcal{E} \int_{\mathcal{D}} \operatorname{trace}(\mathbf{T} \mathbf{L}) d V \mapsto \int_{\mathcal{D}} \operatorname{trace}(\overline{\mathbf{T}} \overline{\mathbf{L}}) d V+\int_{\mathcal{D}} \mathcal{E}\left(\operatorname{trace}\left(\mathbf{T}^{\prime} \mathbf{L}^{\prime}\right)\right) d V$

Then, for covariance of the theory, there must be, for all " $i$ ", the equality: $\Sigma\left(A_{i}\right)-\Sigma\left(A_{i}^{\prime}\right)=0$. For this equivalence it is found that the constraint:

$$
\int_{\mathcal{D}}\left\langle\left[-\bar{\rho} \overline{\mathbf{a}}+\bar{\rho} \mathbf{f}_{B}^{m}-\mathcal{E}\left(\rho^{\prime} \mathbf{a}^{\prime}\right)+\operatorname{div}(\overline{\mathbf{T}})\right], \mathbf{V}_{T}\right\rangle d V=0
$$

must hold in any inertial frame. Since the vector $\mathbf{V}_{T}$ is an arbitrary constant, the standard global mean linear momentum equation is recovered in the form:

$$
\int_{\mathcal{D}}\left[-\bar{\rho} \overline{\mathbf{a}}+\bar{\rho} \mathbf{f}_{B}^{m}-\mathcal{E}\left(\rho^{\prime} \mathbf{a}^{\prime}\right)+\operatorname{div}(\overline{\mathbf{T}})\right] d V=0
$$

Finally, the localization theorem provides the local equation of mean linear momentum in the form:

$$
-\bar{\rho} \overline{\mathbf{a}}+\bar{\rho} \mathbf{f}_{B}^{m}-\mathcal{E}\left(\rho^{\prime} \mathbf{a}^{\prime}\right)+\operatorname{div}(\overline{\mathbf{T}})=0
$$

which is, of course, just the standard Reynolds decomposition of equation (7a) when the body force does not possess a fluctuating component.

The remaining terms on the right hand side of the $\left(A_{i}^{\prime}\right)$ contributions, do however, provide turbulence contributions to the mean total energy equation. Only the mean velocity transformation in equation (10) contains the boost velocity $\mathbf{V}_{T}$.

A deeper understanding of the covariant structure is lacking without a reason for the existence of inertial frames. The principle of Mach was intended to provide some understanding of inertial frames and their role in Newtonian theory. However, as outlined in Moulden [5], the principle of Mach only provides an alternative representation of this background in terms of an integral over the entire Newtonian universe. The principle of Mach adds no fuller explanation of inertia than what Bondi [8] describes as: "... magnitude of the inertia of any body is determined by the masses of the universe and by their distribution". A descriptive comment rather than an explanation of the Mach principle. Einstein had other things to say in response to the ideas of Mach.

For reference, note that the sum $\Sigma_{i}\left(A_{i}\right)$ gives (with the mean motion equation (12) subtracted) the Reynolds decomposition of the energy equation in the form:

$$
\begin{aligned}
\int_{\mathcal{D}}\left[\bar{\rho} \frac{d \bar{e}}{d t}\right. & +\operatorname{trace}(\overline{\mathbf{T}} \overline{\mathbf{L}})+\bar{\rho} \operatorname{div}(\overline{\mathbf{q}})+\langle\operatorname{div}(\overline{\mathbf{T}}, \overline{\mathbf{v}}\rangle+\langle\bar{\rho} \overline{\mathbf{a}}, \overline{\mathbf{v}}\rangle \\
& +\left\langle\bar{\rho} \mathbf{f}_{B}^{m}, \overline{\mathbf{v}}\right\rangle+\mathcal{E}\left\langle\rho^{\prime} \mathbf{a}^{\prime}, \overline{\mathbf{v}}\right\rangle+\mathcal{E}\left\langle\rho^{\prime} \mathbf{f}_{B}^{m}, \mathbf{v}^{\prime}\right\rangle+\mathcal{E}\left\langle\operatorname{div}\left(\mathbf{T}^{\prime}\right), \mathbf{v}^{\prime}\right\rangle \\
& -\left[\mathcal{E}\left\langle\rho^{\prime} \mathbf{a}^{\prime}, \mathbf{v}^{\prime}\right\rangle+\mathcal{E}\left\langle\bar{\rho} \mathbf{a}^{\prime}, \mathbf{v}^{\prime}\right\rangle+\mathcal{E}\left\langle\rho^{\prime} \overline{\mathbf{a}}, \mathbf{v}^{\prime}\right\rangle\right] \\
& \left.+\mathcal{E}\left(\rho^{\prime} \frac{d e^{\prime}}{d t}\right)+\mathcal{E} \operatorname{trace}\left(\mathbf{T}^{\prime} \mathbf{L}^{\prime}\right)+\mathcal{E}\left(\rho^{\prime} \operatorname{div}\left(\mathbf{q}^{\prime}\right)\right)\right] d V=0
\end{aligned}
$$

The localization theorem provides the obvious partial differential equation which need not be recorded herein. Equations (12) and (13) take on their simplest form if the Favre average notation is adopted, but this will not be done herein. The result 
in equation (13) is independent of any turbulence model that may be introduced and, of course, is of no practical use until such a model is included. The above deliberations have shown that:

Lemma 3.2: Consistency,

The Reynolds decomposition commutes with the covariant structure of Newtonian continuum thermomechanics.

to conclude the covariance study of the classical Reynolds decomposition. It only remains to ensure that each of the turbulence models adopted in the applications have the appropriate covariance structure to be consistent with the mean motion equations.

\section{Extensions?}

One of the important practical problems of interest concerns a body moving in a turbulent fluid and, as such, cannot be treated within the framework of Galilean transformations since fluctuations in both orientation and velocity would be required of any coordinate frame attached to the body. Such a coordinate frame would not be inertial and equation (1) would no longer be appropriate. In addition, fluctuations in the body force must also be included in this extended theory. Since Newtonian mechanics is not Euclidean invariant such extended equations, containing these additional fluctuations, cannot be considered within the present framework.

\section{Final remarks}

It has been shown that the Reynolds decomposition process commutes with Galilean transformations in the covariant formulation of Newtonian continuum mechanics contained in axiom 2.0 above. The present study draws attention to the consistency of the Reynolds decomposition in the covariant formulation.

The insistence upon a covariant structure as the basis of classical mechanics, places the first axiom of Newton in a much deeper role than is often portrayed in the standard literature on mechanics. In some sense, this importance is philosophical rather than practical. However, a good theory, must have a sound philosophical background.

The above comments do not, in any way, explain axiom 1 of Newton.

\section{References}

[1] Moulden, T. H. Uniqueness theorems in turbulence models. Proc. 29th ISTS Conference, Nagoya, Japan, 2013

[2] Noll, W. La Méchanique classique, basée sur un axiome d'object-ivité. In: Colloque International, Paris 1959; pp 47-56, 1963

[3] Green, A. E. and Rivlin, R. S. On Cauchy's equations of motion. ZAMP. 15, pp 290-292, 1964 
[4] Moulden, T. H. On the first axiom of Newtonian continuum mechanics. IJPAM, 77 \#2 pp 199-212, 2012

[5] Moulden, T. H. The principle of Mach in Newtonian continuum thermomechanics. IJPAM, vol. 91, No. 4, pp 483-493, 2014

[6] Speziale, C. G. Invariance of turbulent closure models. Phys. Fluids, 22 pp 1033-1037, 1979

[7] Newton, I. Philosophice Nateralis Principia Mathematica. Guil. and Jon. Innys, Regix Societatis Typographos, 3rd Edition, 1726

[8] Bondi, H. Cosmology. Cambridge University Press, 1952 